\title{
ЭКСПЕРИМЕНТАЛЬНЫЕ ИССЛЕДОВАНИЯ УСТОЙЧИВОСТИ ОСЛАБЛЕННЫХ КРУГОВЫМИ ОТВЕРСТИЯМИ ЦИЛИНДРИЧЕСКИХ ОБОЛОЧЕК ПРИ ОСЕВОМ СЖАТИИ
}

Представлены результаты системного экспериментального исследования докритического состояния, закритических форм равновесия и критических нагрузок цилиндрических оболочек с регулярно расположенными в одном поясе среднего поперечного сечения круговыми отверстиями при осевом сжатии. Количество отверстий и их размеры изменялись в широких диапазонах. Испытаны двенадцать серий моделей. Для каждой серии оболочек суммарная площадь всех отверстий оставалась постоянной. Результаты представлены в виде графиков, таблиц и картин форм потери устойчивости.

Ключевые слова: цилиндрическая оболочка; круговые отверстия; осевое сжатие; устойчивость; экспериментальное исследование.

Введение. Тонкостенные цилиндрические оболочки находят широкое применение в ракетной, космической, авиационной, нефтеперерабатывающей, химической и других отраслях. Такие оболочечные элементы зачастую имеют целый ряд отверстий различного количества, фоормы, размеров и расположения, обусловленных конструктивными и технологическими требованиями.

Применение теоретических методов и современных численных средств анализа позволяет достаточно успешно исследовать напряженно-деформированное состояние таких тонкостенных деформируемых систем [5, 11, 15, 16, 20]. Что касается задач устойчивости, то достоверность полученных таким образом решений часто требует дополнительного обоснования. Дело в том, что наличие отверстий в гладкой цилиндрической оболочке приводит к существенному изменению напряженно-деформированного состояния. Наряду с основным напряженным состоянием, которое распространяется по всей поверхности оболочки, вокруг отверстий возникают области концентрации напряжений локального характера, которые зачастую и инициируют локальную потерю устойчивости оболочки в районе отверстий, которая сопровождается образованием больших деформаций и существенным изменением поля напряжений. Как правило, после локальной потери устойчивости оболочка может и далее воспринимать возрастающую нагрузку вплоть до общей потери устойчивости. Изменение геометрии и напряженно-деформированного состояния в процессе локальной потери 
устойчивости носит нелинейный характер, поэтому теоретически определить достоверную величину критической нагрузки, соответствующей общей потере устойчивости оболочки, часто не представляется возможным. В этих условиях чрезвычайно важными являются экспериментальные методы исследования. В тоже время корректное проведение таких исследований связано со значительными трудностями технического характера. Эти обстоятельства обуславливают актуальность экспериментальных исследований этой проблемы, учитывая, что обеспечение устойчивости и несущей способности таких тонкостенных оболочек при действии на них различных внешних нагрузок является одной из важнейших инженерных задач.

Одной из первых статей, посвященных экспериментальным исследованиям влияния отверстий на устойчивость оболочек при осевом сжатии, была работа R. Tennyson [27], который проводил исследование оболочек, ослабленных в средней части цилиндрической поверхности одним круговым отверстием различного диаметра. Оболочки изготавливались методом центробежного литья из эпоксидного материала.

В [10] представлены результаты экспериментального исследования устойчивости круговых продольносжатых цилиндрических оболочек, ослабленных в средней части одним круговым вырезом, при действии осевого сжатия, кручения, внешнего давления и комбинации этих нагрузок. Эксперименты проводились на оболочках, изготовленных из триацетатной плёнки. Проведен анализ зависимости докритического поведения, критических нагрузок и закритического волнообразования от размеров отверстия. В работе Д. Г. Старнса [18] обсуждаются результаты экспериментального исследования влияния круговых вырезов на устойчивость тонких цилиндрических оболочек, изготовленных из майларовой полистероидной плёнки при действии осевой сжимающей силы, изгиба и кручения.

Работа [2] посвящена экспериментальному исследованию устойчивости цилиндрических и конических оболочек с одним и двумя отверстиями круговой и квадратной формы при осевом сжатии. Был сделан вывод, что критические нагрузки для круговых отверстий немного превышают соответствующие нагрузки для квадратных, а несущая способность оболочки с одним круговым или квадратным отверстием меньше несущей способности оболочек с двумя такими отверстиями. Этот результат в полной мере соответствует фризике процесса, поскольку при наличии одного отверстия на поверхности оболочки при ее продольном сжатии появляется изгибающий момент от внецентренного сжатия [7].

В $[8,9,14]$ приводятся результаты экспериментального исследования влияния количества равномерно расположенных в окружном направлении в различных сечениях по длине оболочки круговых отверстий различных размеров на величину несущей способности цилиндрических оболочек при осевом сжатии. Исследовались закритические формы равновесия оболочек с увеличением количества отверстий. В [7] представлены результаты системных экспериментальных испытаний 
моделей продольно сжатых оболочек с одним или несколькими отверстиями квадратной и круговой формы, различных размеров и количества.

В целом, исследованию устойчивости тонкостенных оболочек с круговыми отверстиями при действии как осевой сжимающей силы, так и других видов нагружения посвящено значительное количество работ, среди которых следует отметить публикации $[3,4,6,12,13,17,21$ - 24 , 26, 28].

Модели для испытаний. В качестве материала для изготовления моделей была выбрана чертежная бумага марки «В» ГОСТ 597-73. Впервые возможность качественного исследования устойчивости моделей цилиндрических оболочек, изготовленных из бумаги, была показана Р.В. Роде и Е.Е. Лундквистом [25], а позже В.М. Чебановым [19]. В настоящей статье использована методика изготовления и испытания моделей цилиндрических оболочек из чертёжной бумаги, разработанная Е.Ф. Прокопало [4, 7 -9, 14, 21], где приведено также обоснование корректности использования такого материала. Показано, что этот материал обладает достаточно стабильными механическими характеристиками, ортотропией механических свойств и высокой технологичностью при изготовлении моделей. Более того, невзирая на сравнительно низкие значения упругих характеристик бумаги, по сравнению с металлами, отношение предела текучести этого материала к модулю упругости $\left(\sigma_{\top} / E\right)$ соразмерно с этими данными для листовой нержавеющей стали, что даёт возможность проводить широкомасштабные экспериментальные исследования, которые практически невозможно выполнить на металлических оболочках.

В результате предварительных исследований, выполненных как на плоских образцах, так и на оболочках при помощи электромеханической системы, были определены основные механические характеристики материала: модули упругости $E_{x}=6,9 \cdot 10^{9} \Pi a, E_{y}=3,45 \cdot 10^{9} \Pi a$, $G=1,92 \cdot 10^{9}$ Па и коэфффициенты Пуассона $\mu_{x}=0,3, \mu_{y}=0,15$. Здесь индексы $\mathrm{X}, \mathrm{Y}$ соответствуют главным направлениям ортотропии бумаги. Толщина листа составляла $h=0,23 \cdot 10^{-3}$ . Эта величина была практически неизменной для соответствующей партии бумаги. Измерение толщины листа проводилось при помощи индикатора часового типа с ценой деления шкалы $10^{-6} \mathrm{M}$.

Для изготовления оболочек из листа ватмана вырезались прямоугольные заготовки таким образом, чтобы их стороны были параллельны главным направлениям ортотропии. Направление $E_{x}$ всегда совпадало с направлением образующей оболочки, а $E_{y}-c$ eе направляющей. На заготовках при помощи высечек пробивались круговые отверстия, регулярно расположенные вдоль направляющей симметрично относительно среднего поперечного сечения. Размеры отверстий варьировались в широких диапазонах. Такая технология обеспечивала в районе отверстий отсутствие остаточных деформаций. Затем заготовка склеивалась на металлическом цилиндре. Ширина клеевого шва со- 
ставляла $5 \cdot 10^{-3}$ м. Внутренний радиус всех моделей $-R=37,5 \cdot 10^{-3} M$, рабочая длина $L=75 \cdot 10^{-3} \mathrm{M}$. Полная длина оболочки была принята $L_{п}=115 \cdot 10^{-3} M$. Для обеспечения возможности равномерной передачи на оболочку осевых сжимающих усилий к ее криволинейным кромкам приклеивались металлические торцевые приспособления, имеющие круговое поперечное сечение диаметром $d=75 \cdot 10^{-3} \mathrm{M}$. Ширина приклея составляла $2 \cdot 10^{-2}$ м Такое закрепление краев обеспечивало граничные условия, близкие к жесткому защемлению.

Таблица 1 - Геометрия моделей

\begin{tabular}{|c|c|c|c|c|c|c|c|c|c|c|c|c|c|}
\hline \multirow{2}{*}{$\begin{array}{c}\text { № } \\
\text { Серии }\end{array} \begin{array}{c}\text { Суммарная } \\
\text { относитель- } \\
\text { ная площадь } \\
\text { отверстий F/n }\end{array}$} & \multicolumn{10}{|c|}{ Диаметр отверстий d (мм) } \\
\hline 1 & $\bar{F}_{1}=0$ & - & - & - & - & - & - & - & - & - & - & - & - \\
\hline 2 & $\bar{F}_{2}=0,0043$ & 10 & 7 & 5,5 & 5 & - & 4 & - & - & - & - & - & - \\
\hline 3 & $\bar{F}_{3}=0,0055$ & 11,1 & 8 & 6,4 & 5,5 & 5 & - & - & 4 & - & - & - & 3,2 \\
\hline 4 & $\bar{F}_{4}=0,011$ & 15,8 & 11,1 & 9 & 8 & 7 & 6,4 & 6 & 5,5 & - & 5 & 4,8 & - \\
\hline 5 & $\bar{F}_{5}=0,014$ & 18 & 12,7 & - & 9 & 8 & - & - & 6,4 & 6 & - & 5,5 & - \\
\hline 6 & $\bar{F}_{6}=0,022$ & 22 & 15,5 & 12,7 & 11 & - & 9 & - & - & - & 7 & - & 6,4 \\
\hline 7 & $\bar{F}_{7}=0,028$ & 25 & 18 & - & 12,5 & 11,1 & - & 9,5 & 9 & - & 8 & - & - \\
\hline 8 & $\bar{F}_{8}=0,042$ & 30 & 22 & - & 15,5 & 14 & 12,5 & - & 11 & - & 9,5 & 9 & - \\
\hline 9 & $\bar{F}_{9}=0,07$ & 40 & - & - & 20 & 18 & 16 & 15 & 14 & - & 12,7 & 12,5 & 11,1 \\
\hline 10 & $\bar{F}_{10}=0,11$ & 50 & - & - & 25 & 22 & 20 & 19 & 17,5 & - & 15,8 & 15 & - \\
\hline 11 & $\bar{F}_{11}=0,16$ & 60 & - & - & 30 & - & 25 & 22 & - & 20 & 19 & - & - \\
\hline 12 & $\bar{F}_{12}=0,21$ & 70 & 50 & 40 & - & 30 & - & - & - & - & - & - & - \\
\hline
\end{tabular}

Всего было испытано 12 серий оболочек, общим количеством 160 моделей. Оболочки первой серии были сплошными (4 шт.). В табл. 1 для оболочек всех серий приведены: количество отверстий (n шт.), их диаметр $(d, m M)$ и относительная суммарная площадь круговых отверстий, где $\bar{F}$ равно отношению суммарной площади всех отверстий, вырезанных на конкретной модели к рабочей площади боковой поверхности сплошной оболочки. Каждая номинально геометрически одинаковая модель изготавливалась в двух экземплярах. В каждой серии при различных размерах и количестве отверстий их суммарная площадь оставалась постоянной. На одной оболочке все отверстия были одного размера. 
Методика проведения эксперимента. Испытания проводились на специальной установке, позволяющей нагружать оболочку осевой сжимающей силой (рис. 1). Оболочка (1) нижним торцем крепилась на горизонтальной плите (2), а к верхнему торцу (4) оболочки для передачи

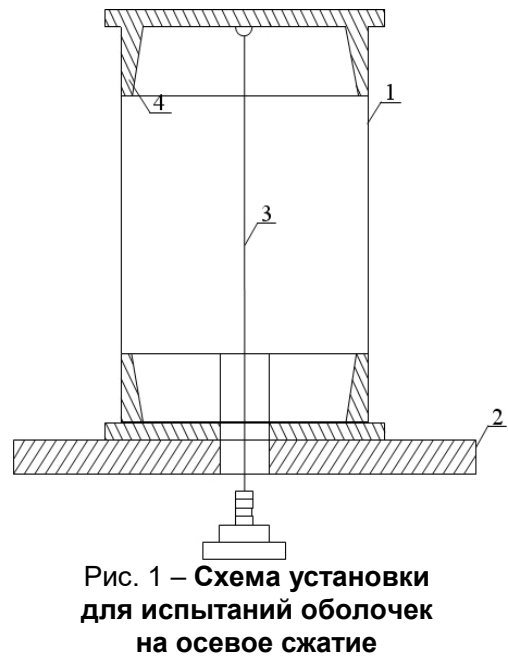

сжимающей силы шарнирно крепилась тяга (3), которая проходила через отверстие в нижнем торце и в плите. Нагружение проводилось через тягу поэтапно по схеме «мертвого» груза при помощи стандартных гирь второго класса точности. В процессе нагружения перемещение груза ограничивалось упором, предотвращающим полное разрушение оболочки после потери её устойчивости. Равнодействующая сжимающего усилия всегда проходила через геометрические центры верхнего и нижнего торцов. При нагружении оболочки верхний торец мог свободно поворачиваться в вертикальной плоскости.

При наличии в оболочке одного отверстия такая схема нагружения приводит к внецентренному сжатию. Для сплошной оболочки и для оболочек с отверстиями, при их количестве, отличном от единицы, и условии их регулярного размещения в одном поперечном сечении, такая схема нагружения отвечает осевому сжатию. Момент потери устойчивости определялся визуально. Оболочки, которые имели локальные формы потери устойчивости, догружались до общей потери устойчивости, которая сопровождалась исчерпанием несущей способности.

Докритическое поведение и закритические формы равновесия. На оболочках с отверстиями диаметром $d=4-6$ мм в докритической стадии изгибающих деформаций вблизи отверстий визуально не наблюдалось. На оболочках с отверстиями диаметром от 8 до 20 мм при нагружении осевой сжимающей силой наблюдался изгиб на краях отверстий, в результате которого образовывались выпучины, направленные от центра кривизны. Они начинались и далее имели максимальное значение на противоположных концах диаметра отверстия, лежащего в среднем поперечном сечении оболочки. Вдоль направляющей протяженность выпучин была сопоставима по размеру с диаметром отверстия. В радиальном направлении максимальные перемещения имели порядок нескольких толщин оболочки на краю отверстия и быстро убывали вдоль образующей. На расстояниях, равных примерно половине радиуса отверстия, с обеих сторон от "вершины" каждого прогиба они принимали отрицательные значения. 


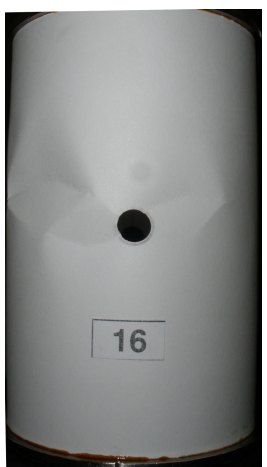

Рис. 2. - Локальная форма потери устойчивости оболочки с отверстием $d=8$ мм
При дальнейшем увеличении нагрузки в этих зонах происходила локальная потеря устойчивости, проявлявшаяся на оболочках при определенном значении внешней нагрузки в зависимости от количества и размера отверстий, с образованием двух-трёх вмятин, которые располагались симметрично или кососимметрично по образующей, проходящей через центр отверстия (рис. 2).

После локальной потери устойчивости были возможны три варианта поведения оболочек: 1) сразу же после локальной потери устойчивости происходила общая потеря устойчивости; 2) происходила локальная потеря устойчивости, далее при неизменной нагрузке вмятины увеличивались и через некоторое непродолжительное время происходила общая потеря устойчивости; 3) после локальной потери устойчивости оболочки несли возрастающую нагрузку, локальные вмятины при этом увеличивались, и в какой-то момент хлопком наступала общая потеря устойчивости, которая сопровождалась исчерпанием несущей способности модели. Превышение нагрузки, соответствующее общей потере устойчивости над локальной, достигало $12 \%$. На оболочках с большим количеством отверстий $n>6$ или с отверстиями большого диаметра $d>20$ мм локальная потеря устойчивости не наблюдалось.

Закритические формы равновесия в основном наследовали картины прогибов докритического поведения оболочки.

Для сплошных оболочек (І серия) потеря устойчивости сопровождалась хлопком с образованием одного-двух поясов ромбовидных вмятин. Количество вмятин по дуге оболочки, как это и следовало ожидать из классических решений [1], было равно шести.

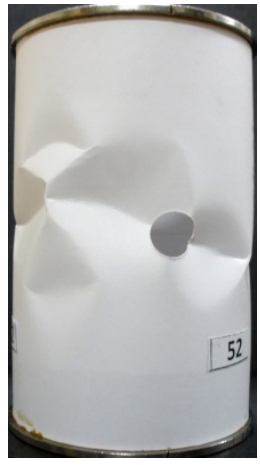

a)

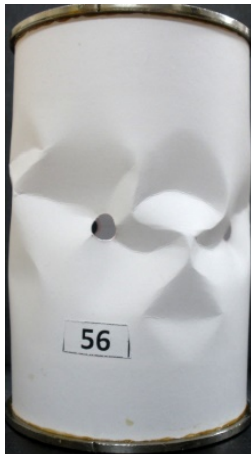

б)

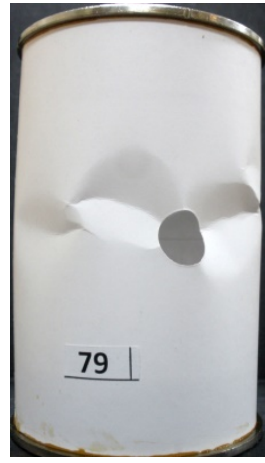

В)

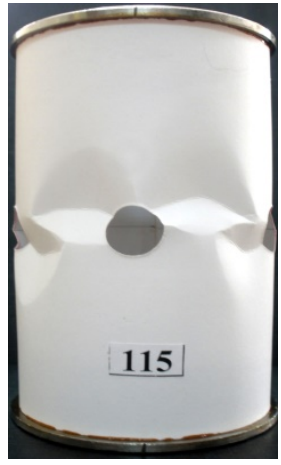

г)

Рис.3 - Формы потери устойчивости цилиндрической оболочки при:

a) $\mathrm{n}=2, d=12,7$ мм; б) $\mathrm{n}=5, d=8$ мм; в) $\mathrm{n}=2, d=15,5$ мм; г) $\mathrm{n}=5, d=14$ мм 
Отметим некоторые общие закономерности закритического волнообразования, характерные для оболочек с отверстиями. При малом количестве отверстий $(\mathrm{n} \leq 5)$, с диаметром не превышающем $d \leq 20$ мм, потеря устойчивости сопровождалась появлением, как правило, нерегулярных вмятин. При этом наибольшие из вмятин проходили через центры отверстий (рис. 3).

Оболочки с одним отверстием всегда теряли устойчивость по несимметричной форме (рис. 4). Увеличение размеров отверстий и их количества приводит к появлению регулярного волнообразования, геометрия которого определяется количеством и размером отверстий.

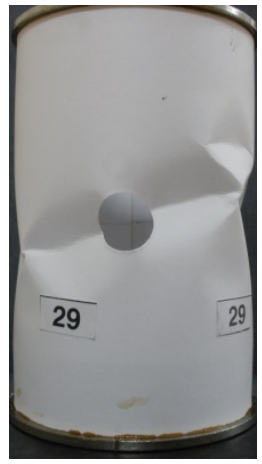

a)

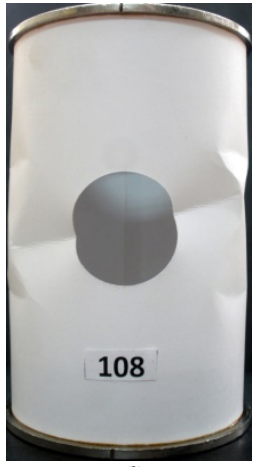

б)

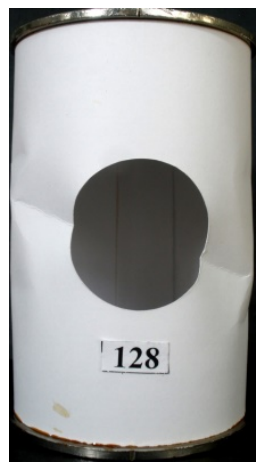

B)

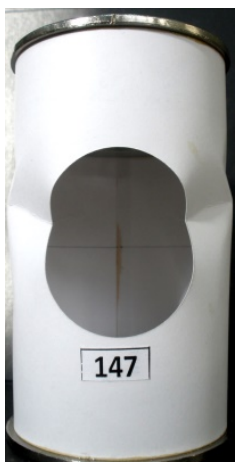

г)

Рис. 4 - Несимметричные формы потери устойчивости оболочки с одним отверстием: а) $d=16$ мм; б) $d=30$ мм; в) $d=40$ мм; г) $d=50$ мм

Для оболочек с шестью отверстиями, при их диаметре, изменяющимся от $d=4$ мм до $d=18$ мм, два соседние пояса вмятин, расположенные симметрично относительно среднего поперечного сечения, образовывали один пояс «сдвоенных» вмятин, в центре каждой из которых находилось отверстие. Овалообразные вмятины были вытянуты кососимметрично относительно образующей, проходящей через центр отверстий (рис. 5).

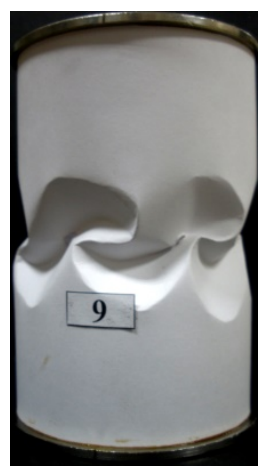

a)

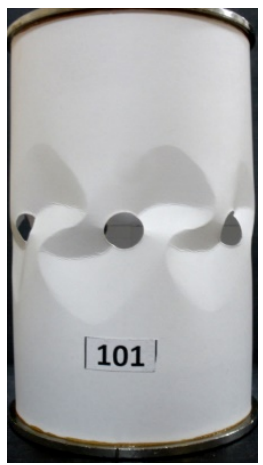

б)

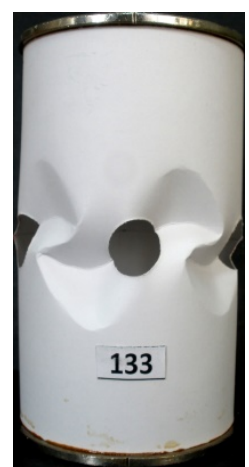

в)

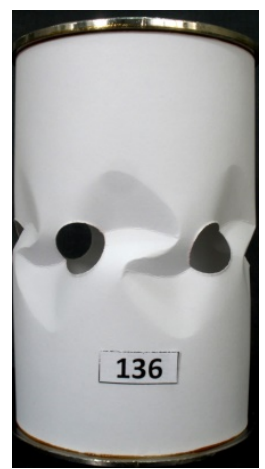

r)

Рис. 5 - Формы потери устойчивости с поясом сдвоенных вмятин $(\mathrm{n}=6)$ : а) $d=4$ мм; б) $d=9,5$ мм; в) $d=18$ мм; г) $d=16$ мм 
Оболочки с отверстиями диаметром $d=8-14$ мм, при их количестве $n=8 \div 10$ теряли устойчивость с образованием одного - двух поясов, в каждом из которых было по пять - шесть вмятин, соответственно. При этом каждая вмятина как бы «опиралась» своими краями на два соседних отверстия (рис. 6).

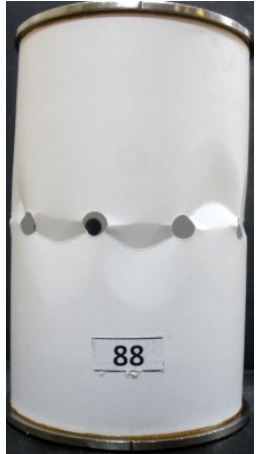

a)

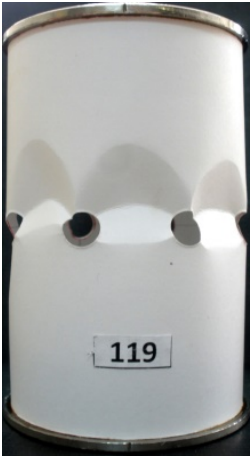

б)

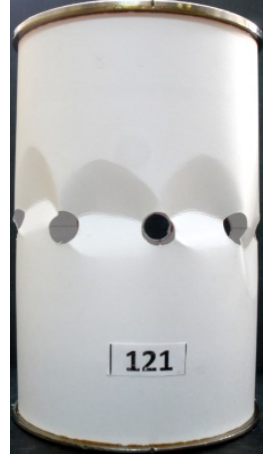

B)

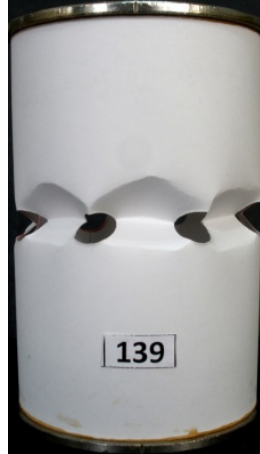

г)

Рис. 6 - Регулярные формы потери устойчивости с образованием вмятин между отверстиями:

а) $\mathrm{n}=10, d=8$ мм; б) $\mathrm{n}=8, d=12$ мм; в) $\mathrm{n}=9, d=10$ мм; г) n=8, $d=14$ мм

С увеличением размеров и количества отверстий в некоторый момент времени потеря устойчивости принимала «панельную» форму (рис. 7). Прогибы оболочки, соответствующие направлению ее перемещений в области волнообразования были направлены либо к центру, либо от центра кривизны. Характерной особенностью такого волнообразования является то, что по длине оболочки оно распространяется на величину, равную приблизительно диаметру отверстия.

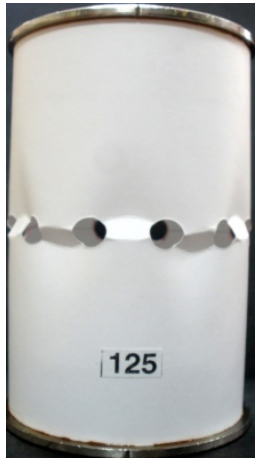

a)

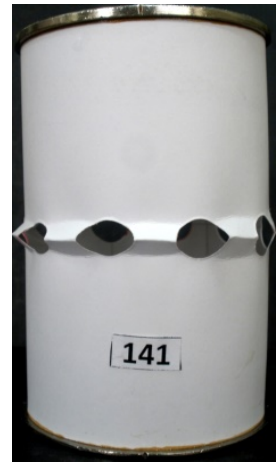

б)

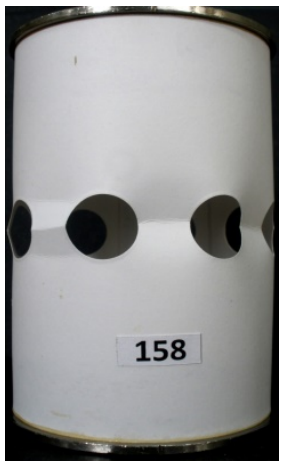

B)

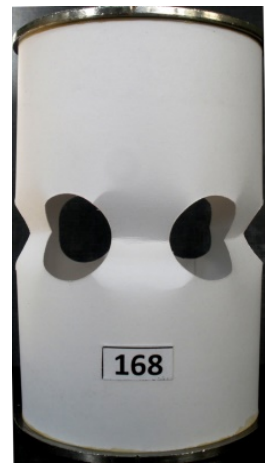

г)

Рис. 7 - «Панельные» формы потери устойчивости:

a) $n=12, d=9$ мм; б) $n=10, d=12,7$ мм; в) $n=8, d=17,5$ мм; г) $n=6, d=25$ мм 
Формы потери устойчивости, как следует из результатов исследований, зависели от количества и размеров отверстий, что вполне объясняется механизмом образования закритических деформаций. Как уже указывалось выше, каждая номинально геометрически одинаковая модель, изготавливалась и испытывалась в двух экземплярах. При этом, значения критических нагрузок, полученные для таких оболочек равной суммарной площадью отверстий, оказались очень близкими для всех моделей всех серий.

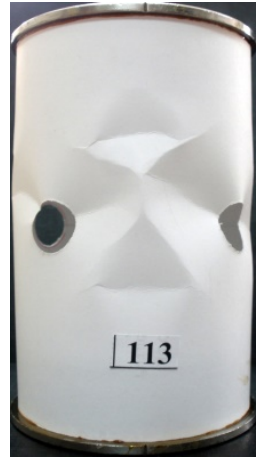

a)

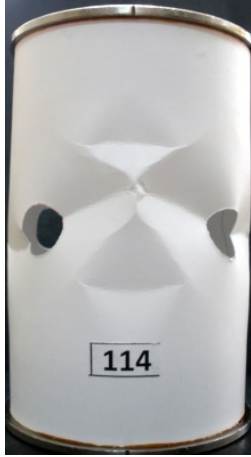

б)

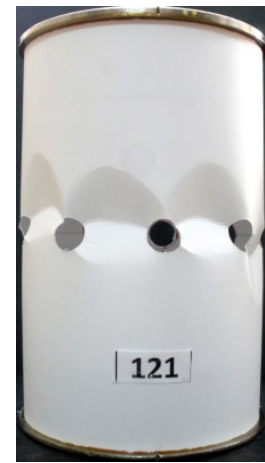

B)

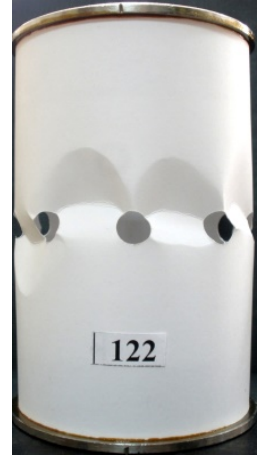

г)

Рис. 8 - Закритические формы равновесия геометрически одинаковых моделей с малыми отверстиями:

а) $\mathrm{n}=4, d=15,5$ мм; б) $\mathrm{n}=4, d=15,5$ мм; в) $\mathrm{n}=9, d=10$ мм; г) $\mathrm{n}=9, d=10$ мм

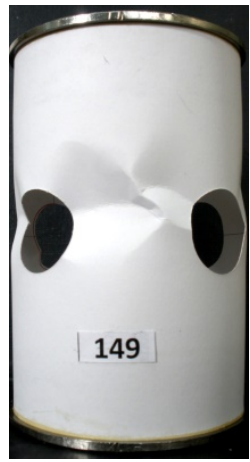

a)

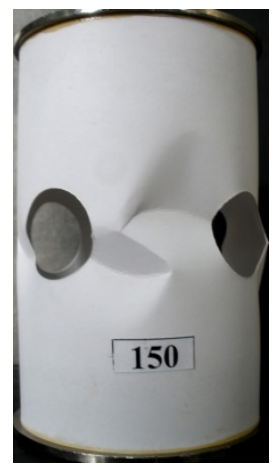

б)

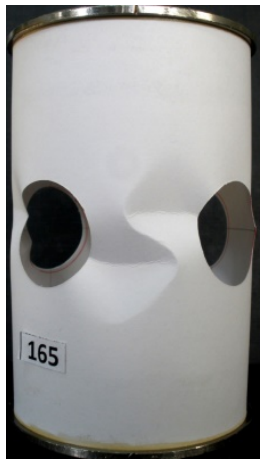

B)

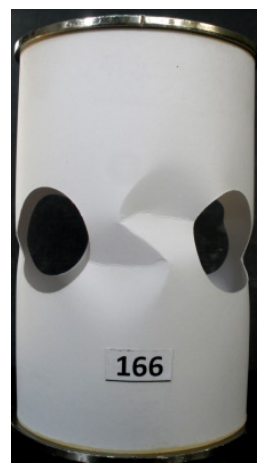

г)

Рис. 9 - Закритические формы равновесия геометрически одинаковых моделей с большими отверстиями:

а) $\mathrm{n}=4, d=25$ мм; б) $\mathrm{n}=4, d=25$ мм; в) $\mathrm{n}=4, d=30$ мм; г) $\mathrm{n}=4, d=30$ мм

Возникает вопрос: будет ли при этом отличаться закритическое волнообразование таких двух геометрически одинаковых моделей? Проведенный анализ показал, что для двух геометрически одинаковых моделей фоормы волнообразования практически совпадают. На рис. 8 и рис. 9 приведены фотографии закритических форм равновесия для 
четырёх пар оболочек. В каждой паре были оболочки геометрически номинально идентичными. Первая пара № 113 и № 114, вторая пара № 121 и № 122, третья пара № 149 и № 150, четвёртая пара № 165 и № 166. Приведенные фотографии свидетельствуют о том, что различие форм закритического волнообразования между двумя оболочками одной пары практически отсутствует. Это позволяет утверждать о несомненно очень высоком качестве проведенного эксперимента.

Анализ экспериментальных результатов. В табл. 2 приведены результаты экспериментальных исследований устойчивости 12-ти серий тонкостенных цилиндрических оболочек, ослабленных круговыми отверстиями общим количеством 160 моделей. Здесь $P$ и $\bar{P}$, соответственно, абсолютные и относительные значения критической силы, а $F$ и $\bar{F}$ - абсолютные и относительные суммарные площади отверстий для каждой серии оболочек. На рис. 10 представлена зависимость усредненных для каждой из двенадцати серий относительных критических нагрузок $\bar{P}=P / P_{0}$ от относительной суммарной площади отверстий на поверхности каждой оболочки; $\bar{F}=F / F_{0}$, где $\mathrm{P}$ - критическая осевая сила для оболочки с отверстиями; $\mathrm{P}_{0}$ - критическая сила для сплошной оболочки; F - суммарная площадь отверстий в каждой оболочке; $F_{0}$ площадь боковой поверхности оболочки. Разбросы критических нагрузок в пределах одинаковых оболочек каждой из серии не превышали 6-8 \%.

Таблица 2 - Абсолютные и относительные значения критической осевой силы и соответствующие им абсолютные и относительные значения суммарной площади отверстий для каждой серии оболочек

\begin{tabular}{|c|c|c|c|c|c|c|c|c|c|c|c|c|}
\hline Серия & 1 & 2 & 3 & 4 & 5 & 6 & 7 & 8 & 9 & 10 & 11 & 12 \\
\hline $\mathrm{P}, H$ & 602 & 480 & 429 & 409 & 400 & 382 & 372 & 324 & 225 & 153 & 105 & 72 \\
\hline $\mathrm{F}, \mathrm{cm}$ & 0 & 0,76 & 0,98 & 1,95 & 2,55 & 3,81 & 4,97 & 7,45 & 12,5 & 19,4 & 28,2 & 37,2 \\
\hline $\bar{P}$ & 1,0 & 0,8 & 0,71 & 0,68 & 0,66 & 0,63 & 0,62 & 0,54 & 0,37 & 0,25 & 0,17 & 0,12 \\
\hline $\bar{F}$ & 0 & 0,0043 & 0,0055 & 0,011 & 0,014 & 0,022 & 0,028 & 0,042 & 0,07 & 0,11 & 0,16 & 0,21 \\
\hline
\end{tabular}

Характерной особенностью полученной зависимости во всём диапазоне изменения суммарной площади отверстий является неравномерное уменьшение критических усилий с увеличением суммарной площади отверстий в каждой оболочке.

При этом, самое интенсивное падение критической нагрузки имеет место на первом участке диаграммы $\bar{P}=f(\bar{F})$, где осевая критическая сила уменьшается примерно на $29 \%$, в то время как суммарная относительная площадь отверстий достигает всего лишь 0,55 \% от площади общей боковой поверхности оболочки. 


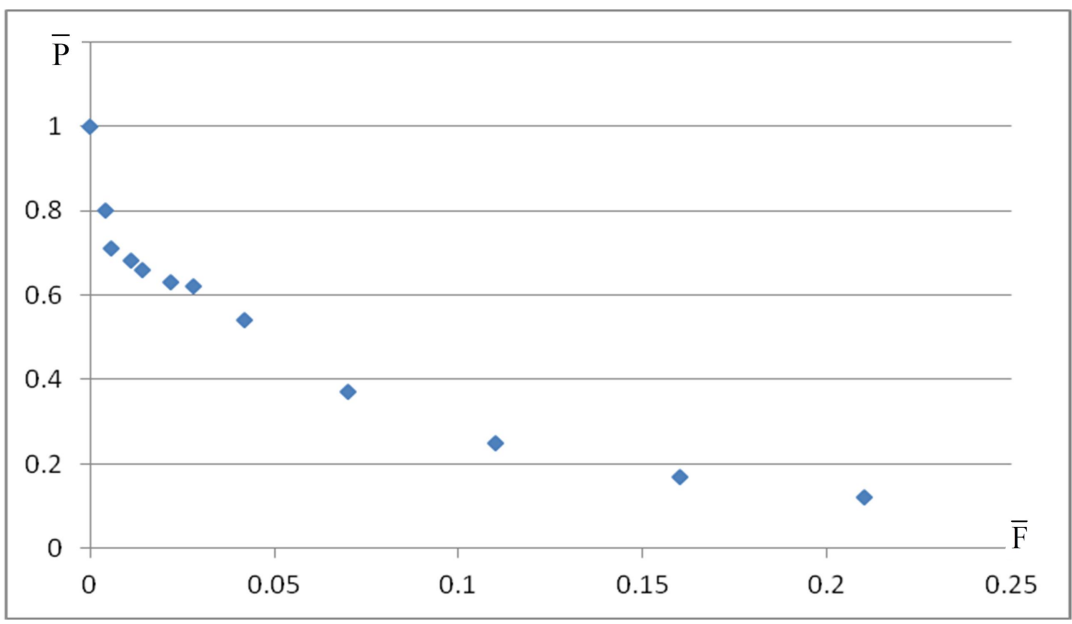

Рис. 10 - Зависимость относительных критических нагрузок $\bar{P}$ от относительной суммарной площади отверстий на каждой оболочке

В дальнейшем интенсивность падения нагрузки существенно уменьшается. Так увеличение параметра $\bar{F}$ (суммарной относительной площади отверстий) от 0,55 \% до $7 \%$ сопровождается уменьшением критической силы на $34 \%$. Таким образом, интенсивность падения нагрузки на втором участке $(0,55 \% \leq \bar{F} \geq 7 \%)$ примерно в десять раз меньше, чем на первом (0\% $\leq \bar{F} \geq 0,55 \%)$.

Выводы. Представлены результаты системного экспериментального исследования устойчивости 160 моделей цилиндрических оболочек при наличии различного количества и диаметра равномерно расположенных в окружном направлении среднего поперечного сечения круговых отверстий при осевом сжатии.

Построены и проанализированы зависимости относительной критической силы от отношения суммарной площади всех отверстий в каждой из оболочек к площади боковой поверхности.

Получены и проанализированы различные формы волнообразования при потере устойчивости в зависимости от количества и размеров отверстий.

Исходя из полученных результатов, можно также сделать вывод, что наличие круговых отверстий, центры которых регулярно расположены в среднем поперечном сечении оболочки с площадью, составляющей приблизительно пятую от часть общей поверхности сплошной оболочки, приводит к уменьшению несущей способности оболочки приблизительно в десять раз.

Показано, что зависимость критической силы потери устойчивости продольно сжатой цилиндрической оболочки от относительной суммарной площади равномерно расположенных в среднем поперечном сече- 
нии круговых отверстий боковой поверхности имеет неравномерный характер.

\section{БИБЛИОГРАФИЧЕСКИЕ ССЫЛКИ}

1. Вольмир А.С. Устойчивость деформируемых систем. М.: Наука, 1967. 984 с.

2. Голда Ю.Л., Преображенский И.Н., Штукарев В.С. Экспериментальное исследование устойчивости оболочек с отверстиями // Прикладная механика. 1973. T. 9. № 1. С. 27-32.

3. Григолюк Э.И., Кабанов В.В. Устойчивость оболочек. М.: Наука, 1978. 360 с.

4. Гудрамович В.С., Дзюба П.А. , Прокопало Е.Ф. Устойчивость ослабленных круговыми отверстиями гладких цилиндрических оболочек при действии поперечного изгиба // Теоретична і прикладна механіка. Донецьк. 2007. Вип. 43. С. 82-87.

5. Гузь А.Н. Концентрация напряжений около отверстий в тонких оболочках // Прикладная механика. 1969. Т. 5. Вып. 3. С. 1-17.

6. Гузь А.Н., Чернышенко И.С., Чехов В.Н. и др. Методы расчёта оболочек: в 5-ти томах. Т. 1 Теория оболочек, ослабленных отверстиями. К.: Наукова думка, 1980. 692 с.

7. Дзюба А.П., Прокопало Є.Ф., Дзюба П.А. Несуча здатність циліндричних оболонок з отворами. Д.: Ліра, 2014. 224 с.

8. Колодяжный А.П., Прокопало Е.Ф. Устойчивость цилиндрических оболочек с круговыми отверстиями при осевом сжатии. - Сообщение 1. Методика проведения и результаты испытаний; - Сообщение 2. Зависимость критической нагрузки от размеров отверстий; - Сообщение 3. Зависимость критической нагрузки от количества отверстий // Theoretical foundations of civil engineering. Warsaw: OWPW. 2010. №18. P. 171-192.

9. Колодяжный А.П., Прокопало Е.Ф. Устойчивость цилиндрических оболочек с системой отверстий при осевом сжатии // Вісник Дніпропетр. ун-ту: Серія «Механіка». 2011. Вип. 15. Т. 2. №. 5. С. 124-132.

10. Коноплев Ю.Г., Тильш А.Л. Устойчивость цилиндрических оболочек с вырезами при осевом сжатии, кручении и внешнем давлении // Теория пластин и оболочек. Казань, 1973. Вып.3. С. 3-13

11. Лурье А.И. Концентрация напряжений в области отверстий на поверхности кругового цилиндра // Прикл. математ. и механ. 1946. Т. Х. Вып. 3. С. 22-30.

12. Моссаковский В.И., Маневич Л.И., Мильцин А.М. Моделирование несущей способности цилиндрических оболочек. К.: Наукова думка, 1977. 136 с.

13. Преображенский И.н. Устойчивость и колебания пластинок и оболочек с отверстиями. М.: Машиностроение, 1981. 192 с.

14. Прокопало Е.Ф., Саверская М. А. Устойчивость цилиндрических оболочек, ослабленных круговыми отверстиями при осевом сжатии // Проблемы вычислительной механики и прочности конструкций: Сб. науч. тр. 2012. Вып.20. С.292-299.

15. Савин Г.Н. Концентрация напряжений около отверстий. К.: Наукова думка, 1968. $887 \mathrm{C}$.

16. Сало В.А., Корниенко А.В. Расчет напряженного состояния упругой оболочки с периодической системой круглых отверстий // Вопросы проектирования и производства конструкций летательных аппаратов. 2011. Вып. 1. С. 76-82.

17. Саченков А.В. Теоретико-экспериментальный метод исследования устойчивости пластин и оболочек // Исследования по теории пластин и оболочек: Сб.научн.тр. Казанского ун-та. 1968. № 6-8. С. 391-433.

18. Старнс Д.Г. Влияние кругового отверстия на устойчивость цилиндрических оболочек при осевом сжатии // Ракетная техника и космонавтика. 1972. Т. 10. № 11. C. 96-104. 
19. Чебанов В.М. Исследование устойчивости тонкостенных оболочек при помощи моделей из бумаги // Инженерный сборник. Институт механики АН СССР. 1955. T. XXII. C. 68-73.

20. Чернишенко І.С., Комарчук С.М., Максимюк В.А., Сторожук Є.А. Моделювання нелінійного деформування ортотропних циліндричних оболонок з отвором при врахуванні ексцентриситету його підкріплення // Доп. Нац. академії наук України. 2016. № 1. С. 34-40.

21. Dzyuba P.A., Prokopalo E.F. Experimental investigation on the torsional stability of cylindrical shells weakened be circular holes // Journal Strength of Materials, November 2017, Vol. 49(6), Iss. 6, P. 829-837.

22. Kamalarajah R., Stoffberg W., Bull J.W., Chizari M. Stress Analysis of Uniform Circular Cylindrical Shells with Large Circular Holes // Proc. of The World Congress on Engineering. London: U.K. 2015., P. 1169-1172

23. Lykhachova O., Krasovsky $V$. Numerical simulation of buckling tests of axially compressed cylindrical shells with one circular cutout (R.Tennyson's experiments) // Theoretical foundations of civil engineering. Warsaw: WP. 2014. № 22. P. 133-136.

24. Maksimyuk V.A., Storozhuк E.A., Chernyshenko I.S. Nonlinear Deformation of Thin Isotropic and Orthotropic Shells of Revolution with Reinforced Holes and Rigid Inclusions // Int. Appl. Mech. 2013. Vol. 49. No 6. P. 685-692.

25. Rhode R.V. Lundquist E.E. Strength tests on paper cylinders in compression bending and shear // NASA. Techn. Notc. 1931. № 370.

26. Stasiewics P. Analytical and Experimental Studies of Stability of Cylindrical Shells with a Cut-Out // Mechanics and Mechanical Engineering. 2013, Vol. 17. No. 2. P. 167-176.

27. Tennyson R.C. The effects of unreinforced circular cutouts on the buckling of circular shells under axial compression. // Trans. ASME. 1968. Vol. 90. No 4. P. 541-546.

28. Wang D.F., Cao P.Z. Stability of thin-walled cylindrical shells under combined loading of overall and local axial compression considering weld geometrical imperfection // Engineering Mechanics. 2009. № 26 (8). P. 65-73.

УДК 539.3

$$
\begin{gathered}
\text { П. А. ДЗЮба, канд. ТеХн. НауК } \\
\text { ЕКСПЕРИМЕНТАЛЬНІ ДОСЛІДЖЕННЯ СТІЙКОСТІ } \\
\text { ОСЛАБЛЕНИХ КРУГОВИМИ ОТВОРАМИ ЦИЛІНДРИЧНИХ } \\
\text { ОБОЛОНОК ПРИ ОСЬОВОМУ СТИСНЕННІ }
\end{gathered}
$$

Подані результати системного експериментального дослідження докритическої поведінки, закритичних форм рівноваги і критичних навантажень циліндричних оболонок з регулярно розташованими в одному поясі середнього поперечного перерізу круговими отворами при осьовому стисненні. Кількість отворів i їх розміри змінювалися в широких диапазонах. Випробувані дванадцять серій моделей. Для кожної серії оболонок сумарна площа всіх отворів лишалася незмінною. Результати подані у вигляді графіків, таблиць і картин форм втрати стійкості.

Ключові слова: циліндрична оболонка; кругові отвори; осьовий стиск; стійкість; експериментальне дослідження.

Забезпечення стійкості і несучої здатності тонкостінних оболонок 3 отворами при дії на них різних зовнішніх навантажень є однією з найважливіших інженерних задач. 
Застосування теоретичних методів і сучасних числових засобів аналізу дозволяє досить успішно досліджувати напружено-деформований стан таких тонкостінних деформованих систем. В задачах стійкості достовірність отриманих таким чином рішень часто вимагає додаткового обґрунтування. Це пов'язано з тим, що наявність отворів в гладкій циліндричній оболонці призводить до істотної зміни напруженодеформованого стану в процесі ії навантаження.

Поряд з основним напруженим станом, що поширюється по всій поверхні оболонки, навколо отворів виникають області концентрації напружень локального характеру, які найчастіше і ініціюють локальну втрату стійкості оболонки в околі отворів, яка супроводжується утворенням великих деформацій і істотною зміною поля напружень.

Зміна геометрії і напружено-деформованого стану в процесі локальної втрати стійкості носять нелінійний характер, тому теоретично визначити достовірну величину критичного навантаження, що відповідає загальній втрати стійкості оболонки, часто не представляється можливим. У цих умовах надзвичайно важливими $є$ експериментальні методи дослідження. У той же час коректне проведення таких досліджень пов'язано зі значними труднощами технічного характеру. Ці обставини обумовлюють актуальність експериментальних досліджень цієї проблеми.

У статті представлені результати системного експериментального дослідження докритического стану, закритичних фрорм рівноваги і критичних навантажень циліндричних оболонок з регулярно розташованими в одному поясі середнього поперечного перерізу круговими отворами при осьовому стисненні. Як матеріал для виготовлення моделей було обрано чертежний папір марки «В». Наведено обґрунтування коректності та ефрективності використання такого матеріалу.

Всього було випробувано 12 серій оболонок, загальною кількістю 160 моделей. Оболонки першої серії були суцільними. Кількість та розміри отворів варіювалися в широких диапазонах. Кожна номінально геометрично однакова модель виготовлялася в двох примірниках. У кожній серії при різних розмірах і різній кількості отворів їх сумарна площа лишалася незмінною. На одній оболонці всі отвори були одного розміру. Випробування проводилися на спеціальній установці, що дозволяє навантажувати оболонку осьової стискальною силою.

Побудовано і проаналізовано залежності осередненних значень критичної сили від сумарної площі отворів в оболонці (відповідно до кожної з дванадцяти серій оболонок).

Отримані картини різних форм хвилеутворення при втраті стійкості в залежності від кількості та розмірів отворів.

Характерною особливістю отриманої залежності критичної сили втрати стійкості поздовжньо стислої циліндричної оболонки від відносної сумарної площі рівномірно розташованих в середньому поперечному перерізі кругових отворів бічній поверхні $є$ нерівномірне зменшення критичних зусиль зі збільшенням сумарної площі отворів на кожній оболонці. 


\section{P. A. Dzyuba, PhD (Tech.) \\ EXPERIMENTAL STABILITY RESEARCHES OF CYLINDRICAL SHELLS WEAKENED BY CIRCULAR HOLES UNDER AXIAL COMPRESSION}

The results of a systematic experimental study of the subcritical behavior, supercritical forms of equilibrium and critical loads of cylindrical shells with circular holes regularly located in one belt, symmetrically relative to the average crosssection, under axial compression are presented. The number of holes and their sizes varied within wide limits. Twelve series of models were tested, for each series of shells the total area of all holes remained constant. The results are presented in the form of graphs, tables and pictures of buckling forms. study.

Keywords: cylindrical shell; circular holes; axial compression; stability; experimental

The results of a systemic experimental study of the subcritical state, supercritical forms of equilibrium and critical loads of cylindrical shells with circular holes regularly located in one belt of the middle cross-section under axial compression are presented. The number of holes and their sizes varied within wide limits.

Drawing paper of "B" type was chosen as a material for making models. The substantiation of the correctness and efficiency of using such a material for the manufacture of models of cylindrical shells is presented in $[4,7,9,10$, $14,19,21,25]$.

In total, 12 series of shells were tested, with a total of 160 models. For each series of shells, the total area of all holes remained constant. The shells of the first series were solid. Each nominally geometrically identical model was made in duplicate. In each series, with different sizes and different numbers of holes, their total area remained constant. On one shell, all the holes were the same size. The tests were carried out on a special installation that allows the shell to be loaded with an axial compressive force.

The dependences of the averaged values of the critical force on the total area of holes on the shell (in accordance with each of the twelve series of shells) are constructed and analyzed.

Pictures of various forms of wave formation with loss of stability are obtained depending on the number and size of holes.

A characteristic feature of the obtained dependence of the critical buckling force of a longitudinally compressed cylindrical shell on the relative total area of uniformly spaced circular holes of the lateral surface in the middle cross-section is an uneven decrease in critical forces with an increase in the total area of holes on each shell.

\section{REFERENCES}

1. Volmir A.S. Stability of deformable systems. Moscow: Nauka, 1967. 984 p. (in Russian). 
2. Golda Yu.L., Preobrazhensky I.N., Shtukarev V.S. Experimental Investigation of the stability of shells with holes // Applied Mechanics. 1973. T. 9. No. 1. P. 27-32. (in Russian).

3. Grigolyuk E.I., Kabanov V.V. The stability of the shells. Moscow: Nauka, 1978. 360 p. (in Russian).

4. Hudramovich V.S., Dzyuba P.A., Prokopalo E.F. Stability of smooth cylindrical shells weakened by circular holes under transverse bending // Theoretical and Applied Mechanics. Donetsk. 2007. Vip. 43. S. 82-87. (in Russian).

5. Guz A.N. Stress concentration near holes in thin shells // Applied Mechanics. 1969. Vol. 5. Iss. 3, P. 1-17. (in Russian).

6. Guz A.N., Chernyshenko I.S., Chekhov V.N. and etc. Methods for calculating shells: in 5 volumes. Vol. 1 Theory of shells weakened by holes. Kiev: Naukova Dumka, 1980. 692 p. (in Russian).

7. Dzyuba A.P., Prokopalo E.F., Dzyuba P.A. The bearing capacity of cylindrical shells with openings. Dnepr: Lira, 2014. 224 p. (in Russian).

8. Kolodyazhny A.P., Prokopalo E.F. Stability of cylindrical shells with circular holes under axial compression. - Message 1. Procedure and test results; - Message 2. Dependence of critical load on hole sizes; - Message 3. Dependence of the critical load on the sizes and number of holes // Theoretical foundations of civil engineering. Warsaw: OWPW. 2010. No. 18. P. 171-192. (in Russian).

9. Kolodyazhny A.P., Prokopalo E.F. Stability of cylindrical shells with a system of holes under axial compression // Bulletin of Dnipropetrovsk University: Series "Mekhanika". 2011. Vol. 2. Iss. 15. No. 5. P. 124-132. (in Russian).

10. Konoplev Yu.G., Tilsh A.L. Stability of cylindrical shells with cutouts under axial compression, torsion and external pressure // Theory of plates and shells. Kazan. 1973. Iss. 3. P. 3-13. (in Russian).

11. Lurie A.I. Concentration of stresses in the area of holes on the surface of a circular cylinder // Applied Mathematics. and mechan. 1946. Vol. X. Iss. 3. P. 22-30. (in Russian).

12. Mossakovsky V.I., Manevich L.I., Miltsin A.M. Modeling the bearing capacity of cylindrical shells. Kiev: Naukova Dumka, 1977. 136 p. (in Russian).

13. Preobrazhensky I.N. Stability and vibrations of plates and shells with holes. Moscow: Mashinostroenie, 1981. 192 p. (in Russian).

14. Prokopalo E.F., Saverskaya M.A. Stability of cylindrical shells weakened by circular holes under axial compression. Problems of computational mechanics and strength of structures.: Collection of scientific articles. 2012. Iss. 20. P. 292-299. (in Russian).

15. Savin G.N. Stress concentration around holes. Kiev: Naukova Dumka, 1968.887 p. (in Russian).

16. Salo V.A., Kornienko A.V. Calculation of the stress state of an elastic shell with a periodic system of circular holes // Issues of design and production of aircraft structures. 2011. Iss. 1. P. 76-82. (in Russian).

17. Sachenkov A.V. Theoretical and experimental method for studying the stability of plates and shells // Research on the theory of plates and shells: Collection of scientific works of Kazan University. 1968. No. 6-8. P. 391-433. (in Russian).

18. Starnes D.G. Influence of a circular hole on the stability of cylindrical shells under axial compression // Rocket technology and cosmonautics. 1972. Vol. 10.No. 11. P. 96104. (in Russian).

19. Chebanov V.M. Investigation of the stability of thin-walled shells using paper models // Engineering collection. Institute of Mechanics of the Academy of Sciences of the USSR. 1955. Vol. XXII. P. 68-73. (in Russian).

20. Chernishenko I.S., Komarchuk S.M., Maksimyuk V.A., Storozhuk E.A. Modeling of nonlinear deformation of orthotropic cylindrical shells with a hole taking into account the 
eccentricity of its reinforcement // Reports of Nat. Academy of Sciences of Ukraine. 2016. No. 1. P. 34-40. (in Ukrainian).

21. Dzyuba P.A., Prokopalo E.F. Experimental investigation on the torsional stability of cylindrical shells weakened be circular holes // Journal Strength of Materials, November 2017, Vol. 49(6). Iss. 6. P. 829-837.

22. Kamalarajah R., Stoffberg W., Bull J.W., Chizari M. Stress Analysis of Uniform Circular Cylindrical Shells with Large Circular Holes // Proc. of The World Congress on Engineering. London: U.K. 2015. P. 1169-1172.

23. Lykhachova O., Krasovsky V. Numerical simulation of buckling tests of axially compressed cylindrical shells with one circular cutout (R.Tennyson's experiments) // Theoretical foundations of civil engineering. Warsaw: WP. 2014. No 22. P. 133-136.

24. Maksimyuk V.A., Storozhuk E.A., Chernyshenko I.S. Nonlinear Deformation of Thin Isotropic and Orthotropic Shells of Revolution with Reinforced Holes and Rigid Inclusions // Int. Appl. Mech. 2013. Vol. 49. No 6. P. 685-692.

25. Rhode R.V. Lundquist E.E. Strength tests on paper cylinders in compression bending and shear // NASA. Techn. Notc. 1931. No. 370.

26. Stasiewics P. Analytical and Experimental Studies of Stability of Cylindrical Shells with a Cut-Out // Mechanics and Mechanical Engineering. 2013. Vol. 17. No. 2. P. 167176.

27. Tennyson R.C. The effects of unreinforced circular cutouts on the buckling of circular shells under axial compression. // Trans. ASME. 1968. Vol. 90. No. 4. P. 541-546.

28. Wang D.F., Cao P.Z. Stability of thin-walled cylindrical shells under combined loading of overall and local axial compression considering weld geometrical imperfection // Engineering Mechanics. 2009. No. 26 (8). P. 65-73.

Днепровский национальный университет имени Олеся Гончара, Днепр, Украина 